\title{
A study on the immediate effects of plantar vibration on balance dysfunction in patients with stroke
}

\author{
Maede Khalifeloo', Soofia Naghdi ${ }^{1,2,3, *}$, Noureddin Nakhostin Ansari ${ }^{1,2,3}$, Mohammad Akbari ${ }^{4}$, Shohreh Jalaie', Davood Jannat ${ }^{5}$, \\ Scott Hasson ${ }^{6}$
}

'Department of Physiotherapy, School of Rehabilitation, Tehran University of Medical Sciences, Tehran, Iran

${ }^{2}$ Sports Medicine Research Center, Neuroscience Institute, Tehran University of Medical Sciences, Tehran, Iran

${ }^{3}$ Neuromusculoskeletal Research Center, Iran University of Medical Sciences, Tehran, Iran

${ }^{4}$ Department of Physical therapy, School of Rehabilitation Sciences, Iran University of Medical Sciences, Tehran, Iran

${ }^{5}$ Industrial Engineering, Tarbiat Modares University, Tehran, Iran

${ }^{6}$ Department of Physical Therapy, Georgia Regents University, Augusta, GA, USA

The aim of this study was to estimate the immediate effects of plantar vibration, applied to the more affected foot, on balance impairment in patients post-stroke. This pretest-posttest clinical study included $18 \mathrm{pa-}$ tients (13 men) poststroke; mean age $56.0 \pm 8.9$ years (range, $41-71$ years). One session of 5-min vibratory stimuli (frequency, $100 \mathrm{~Hz}$ ) was applied to the plantar region of the more affected foot of all participants. The plantar vibration significantly improved the Timed UP and Go test $(P=0.03$, Cohen $d=0.15)$, ankle plantar flexor muscle spasticity $(P=0.008)$, and ankle passive range of motion $(P<0.001$, Cohen $d=0.74)$. The posturography measures and Functional Reach Test did not improve significantly $(P>0.05)$. Vibration stimuli applied to the plantar re- gion of the more affected foot had significant effects on spasticity, ankle passive range of motion and dynamic balance as evaluated by the Timed Up and Go test in patients poststroke. There was no effect on static balance performance. Based on the results, the focal vibratory stimuli applied directly to the plantar region of the more affected foot may be recommended to improve the functional mobility and dynamic balance in patients with stroke.

Keywords: Stroke, Balance, Vibration, Posturography, Spasticity, Physiotherapy

\section{INTRODUCTION}

Stroke is the most common cause of disability in adults. Patients with stroke often experience various problems such as muscle spasticity, weakness, abnormal gait, and balance dysfunction (Arene and Hidler, 2009). Balance impairment is common after stroke which increases the risk of falls (Lamb et al., 2003). A study found that $83 \%$ of patients with stroke had balance dysfunction (Tyson et al., 2006).

Fall is a common complication observed in as high as $73 \%$ of patients with stroke (Yates et al., 2002) which may contribute to serious injuries and life-threatening problems (Divani et al., 2009). Thus, improvement of balance and reducing falls are one of the important goals in stroke neurorehabilitation.

Patients with stroke usually present with sensory deficits and reduction in cutaneous sensation (Carey, 1995; Sterzi et al., 1993). Muscle weakness and sensory dysfunction are factors influencing poor balance in patients poststroke (Tyson et al., 2006). The impairment of plantar cutaneous sensation has been recently reported to contribute to balance dysfunction in individuals after stroke (Parsons et al., 2016). Sensory deficits, therefore, may contribute to poor balance, and can have negative effects on the functional outcomes of patients with stroke.

There are various strategies of physiotherapy (PT) interventions used for treating impaired balance in patients poststroke (Chen and Shaw, 2014; Pollock et al., 2003). PT interventions have
${ }^{\star}$ Corresponding author: Soofia Naghdi (iD https://orcid.org/0000-0002-5062-7747 Department of Physiotherapy, School of Rehabilitation, Tehran University of Medical Sciences Enghelab Ave, Pich-e-shemiran, Tehran 11489, Iran Tel: +98-2177535132, Fax: +98-2177727009, E-mail: naghdi@sina.tums.ac.ir Received: January 18, 2018 / Accepted: February 14, 2018
This is an Open Access article distributed under the terms of the Creative Commons Attribution Non-Commercial License (http://creativecommons.org/licenses/by-nc/4.0/) which permits unrestricted non-commercial use, distribution, and reproduction in any medium, provided the original work is properly cited. 
shown limited effect on improving balance in individuals after stroke (Geurts et al., 2005; Pollock et al., 2014). The evidence for the efficacy of sensory stimulation modalities on balance in patients after stroke is limited (Geiger et al., 2001; Johansson et al., 2001; Pomeroy et al., 2006).

Vibration stimulation is an effective modality which can be used in neurological rehabilitation (Sui et al., 2014). Vibration based methods in either focal or whole body vibration (WBV) form have been used in healthy and patients populations to improve functional outcomes (e.g., Murillo et al., 2014; Schlee et al., 2012; Wanderley et al., 2011). A recent systematic review and meta-analysis included seven studies with 298 chronic stroke patients to assess the effects of WBV and found no beneficial effects of WBV on balance and gait performance (Lu et al., 2015). However, studies on the effects of focal vibration applied to the plantar region in elderly women (Wanderley et al., 2011) and application of vibration to the feet via vibrating insoles in young and elderly participants (Priplata et al., 2003) have demonstrated beneficial effects of plantar vibration on balance control. A case study to demonstrate the short-term effects of plantar vibration in a 72-year-old man with stroke reported beneficial effects on balance (Naghdi et al., 2013). Recently, a study used clinical measure of Mini-Balance Evaluation Systems Test (Mini-BESTest) to investigate the effects of plantar vibration on balance in patients suffering from stroke and found improvements in balance, ankle plantar flexor spasticity, and ankle dorsiflexion passive range of motion (PROM) (Karimi-AhmadAbadi et al., 2017).There are no further studies addressing the effects of plantar vibratory stimulation on balance control in patients poststroke (Murillo et al., 2014). Therefore, the objective of the present study was to assess the effects of focal vibratory stimuli applied to the plantar region on the balance of patients after stroke. We postulated that the balance of patients with stroke could be improved through the application of vibration directly to the affected foot.

\section{MATERIALS AND METHODS}

\section{Design}

A clinical investigation with a pretest-posttest design was followed for this pilot study. The Review Board and the Ethics Committee of Tehran University of Medical Sciences (TUMS) approved the study protocol.

\section{Participants}

Patients with stroke attending the Rehabilitation Clinics of
TUMS were recruited to participate in this study. Inclusion criteria were: (a) first-ever stroke, (b) age $\geq 18$ years, (c) duration since stroke $\geq 6$ months, (d) balance deficit confirmed by one leg stance (OLS) test; patient unable to stand on affected leg for 30s with eyes open, (e) able to walk without the use of aids, and (f) able to understand verbal commands. Exclusion criteria were: (a) participation in other treatment protocols for balance, (b) presence of conditions other than stroke affecting balance, (c) history of lower-limb surgery within the previous year, (d) fixed ankle contractures, and (e) unwillingness for participation in the study. Written informed consent was obtained from all individuals before taking part in the study.

\section{Procedure}

This study was performed in Physiotherapy Clinic for Stroke at School of Rehabilitation, TUMS. The participants were interviewed to collect information including: age, sex, diagnosis (cerebral infarction or hemorrhage), duration since stroke onset, and affected side. The height and weight were measured to calculate the body mass index $\left(\mathrm{BMI}\left[\mathrm{kg} / \mathrm{m}^{2}\right]=\right.$ weight $[\mathrm{kg}] /$ height $\left[\mathrm{m}^{2}\right]$ ). The outcome measures of Timed Up and Go test (TUG), Functional Reach Test (FRT), Modified Modified Ashworth Scale (MMAS), and ankle PROM were administered in this order to participants. The second part of the assessment was posturography using force platform during bipedal standing in the eyes open and closed conditions to calculate the anteroposterior displacements (AP) of center of pressure (COP) in $\mathrm{cm}$, mediolateral (ML) displacements in $\mathrm{cm}$, surface area $\left(\mathrm{cm}^{2}\right)$, and mean velocity $(\mathrm{cm} / \mathrm{sec})$. All measurements were taken before and immediately after treatment. An experienced physiotherapist in neurorehabilitation performed all the assessments.

\section{Outcome measures}

The primary outcome measures were: (a) TUG, (b) FRT, and (c) posturography balance measures. The MMAS and ankle PROM were the secondary outcomes.

\section{Timed Up and Go}

The participant was instructed to sit back in the chair without arms, to stand up on the command "Go," walk $3 \mathrm{~m}$ to the line marked on the floor at a normal pace, turn $180^{\circ}$, and walk back to the chair, and sit down again on the chair (Podsiadlo and Richardson, 1991). The assessor recorded the time with a stopwatch in seconds. The TUG test is a reliable and valid functional test in patients with stroke to monitor changes in mobility and dynamic 
balance after treatment (Flansbjer et al., 2005; Knorr et al., 2010; $\mathrm{Ng}$ and Hui-Chan, 2005).

\section{Functional Reach Test}

The participant was asked to stand with feet shoulder width apart with the affected side next to the wall. From this position, the participant was instructed to clasp their hands and forward flex the arm to approximately $90^{\circ}$ parallel to the floor. Then, the participant was asked to reach forward as far as possible without taking a step or losing balance (Duncan et al., 1990). The FRT has excellent intrarater reliability and validity to evaluate static standing balance in patients with stroke (Duncan et al., 2003).

\section{Modified Modified Ashworth Scale}

Participants were rested for $5 \mathrm{~min}$ in the supine position prior to testing. The physiotherapist used the ordinal MMAS for rating plantarflexor spasticity from 0 (no increase in muscle tone) to 4 (affected part rigid) (Ansari et al., 2006b; Ansari et al., 2008). The MMAS has shown very good intrarater reliability for assessing plantarflexor spasticity (Ghotbi et al., 2011). In this study, the Persian version of MMAS was used (Nakhostin Ansari et al., 2012).

\section{Ankle PROM}

The participant was in supine position on an examination table with the knee in extension (Radinmehr et al., 2017). The $90^{\circ}$ was defined as the neutral position for the measurements using an ankle biplane goniometer (Bissell Health Care, model 7524, USA). Ankle dorsiflexion and plantarflexion from the neutral position was given either a positive or negative value (Ansari et al., 2006a).

\section{Posturography}

A Kistler force platform (Kistler Instrumente AG, Eulachstrasse 22, 8408 Winterthur, Switzerland) was used for postural sway measurements. The participants were asked to stand barefoot on the force platform with heels $9 \mathrm{~cm}$ apart and toes at $30^{\circ}$ out and arms along the body sides. Participants were requested to stand comfortably while gazing at a point placed at a 2-m distance. To collect the COP data, three trials of 20s with eyes open or closed were made with 20 -sec rest interval between trials in seated position. The average of the 3 trials was calculated and used for analysis. The data were collected with a sampling frequency of $100 \mathrm{~Hz}$ and a zerophase, fourth-order Butter worth low-pass filter and $10-\mathrm{Hz}$ cutoff frequency.

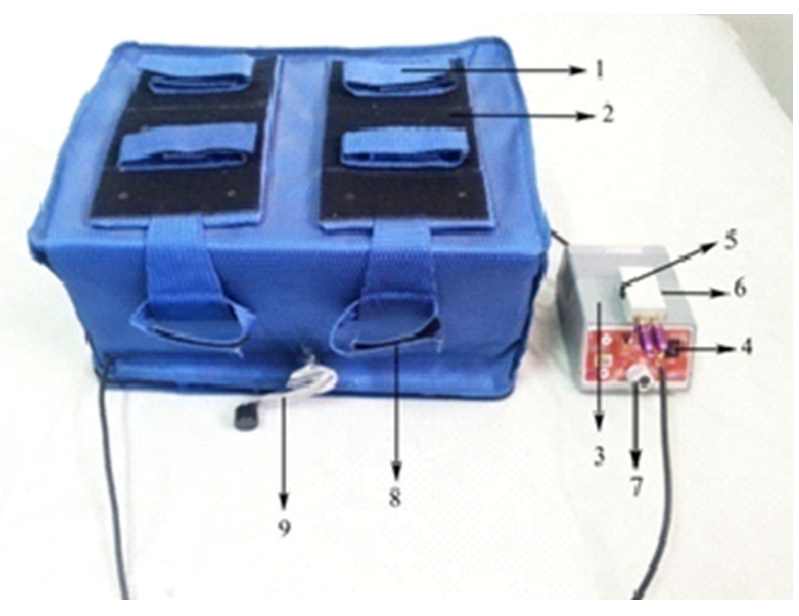

Fig. 1. Plantar vibratory device. (1) Foot strap, (2) foot plate, (3) adaptor, (4) on/ off switch, (5) right on/off switch, (6) left on/off switch, (7) voltage control switch, (8) ankle strap, and (9) plate inclination handle.

\section{Intervention}

A custom-made vibratory stimulation device (Erteashate Tebbie Iranian Co., Tehran, Iran) was used to deliver the vibration stimuli to the plantar region while the participant was in a supine position (Naghdi et al., 2013). The device consisted of two vibrators mounted within a box (dimension [width $\times$ length $\times$ height, $30 \times$ $45 \times 20 \mathrm{~cm}]$ ) (Fig. 1) with the top of box having two plates for each foot through which vibratory stimulations could be delivered to the entire plantar regions. The device delivers vibration stimuli with frequencies ranging from $40-100 \mathrm{~Hz}$, and each foot could be treated separately or simultaneously using this device. Velcro straps were used to fix the feet in place. The angle of the box could be adjusted to the foot position so that the vibratory stimulation could be delivered to the entire plantar region.

A physiotherapist provided the intervention using the vibratory stimulation device. The participant was asked to lie on an examination table in a supine position with knees flexed and the more affected foot on the same plate as the vibratory stimulation device. Participants received vibratory stimulation $(100 \mathrm{~Hz})$ to the plantar region for 5 min to only the more affected foot for a single 5 -min session.

\section{Data analysis}

SPSS ver. 17.0 (SPSS Inc., Chicago, IL, USA) for windows was used to perform statistical analyses. One-Sample Kolmogorov-Smirnov test was used to determine the distribution of sample data. The mean (standard deviation) and median (interquartile range) were calculated for continuous and ordinal variables, respectively. Paired $t$-test was employed to compare the prepost 
data. A General Linear Model, Within Participants Design, was used to determine the time effect (two testing pre- and posttreatment), test condition effect (eyes open, eyes closed), and time $\times$ test condition interaction. The MMAS scores were analyzed by Wilcoxon signed rank test (WSRT). Cohen $d$ was used to measure the treatment effect sizes. $P$-values were set at $\leq 0.05$ defined as statistically significant.

\section{RESULTS}

Eighteen participants with stroke (male, 13; female, 5; mean age, 56.0 \pm 8.9 ; stroke duration, 33.0 28.3 months; BMI $28.0 \pm 5.2 \mathrm{~kg} / \mathrm{m}^{2}$ ) volunteered to participate in the study (Table 1). Ten participants had left hemiplegia. The data was normally distributed for continuous variables, therefore we used parametric tests.

The severity of spasticity statistically improved after treatment (median 3 [1-3] to 2 [1-3]; WSRT, $Z=-2.65, P=0.008$ ). Participants improved in the TUG and FRT after vibration therapy, however only the TUG changes were statistically significant $(P=0.03)$, as demonstrated in Table 2 . The effect sizes for both TUG test and FRT were small (Table 2). The ankle PROM significantly improved after treatment (WSRT, $Z=-3.63, P<0.001$; pre, 4.4 5.2 [range, -5 to 14]; post, 8.3 \pm 5.4 [range, $0-20.0$ ]). The effect size for the ankle PROM was large (Cohen $d=0.74$ ). No significant improvements occurred in the posturography variables with eyes open or closed after vibration therapy (Table 3). Only significant main effect of vision was revealed for mean velocity $(F=44.99, P<0.001), \mathrm{AP}$ and ML displacements $(F=34.75$, $P<0.001)$. The time $\times$ vision interaction was not significant for

Table 1. Demographic characteristics of patients with stroke $(n=18)$

\begin{tabular}{lc}
\hline Characteristic & Mean \pm SD (range) \\
\hline Age $(\mathrm{yr})$ & $56.0 \pm 8.9(41-71)$ \\
Height $(\mathrm{cm})$ & $166 \pm 10(146-190)$ \\
Weight $(\mathrm{kg})$ & $78.2 \pm 18.0(54.5-105.5)$ \\
Body mass index $\left(\mathrm{kg} / \mathrm{m}^{2}\right)$ & $28.0 \pm 5.2(20.5-37.4)$ \\
Duration of stroke $(\mathrm{mo})$ & $33.0 \pm 28.3(6.0-96.0)$ \\
\hline SD, standard deviation. &
\end{tabular}

Table 2. Results of clinical tests

\begin{tabular}{lcccc}
\hline Variable & Pre & Post & P-value & Cohen d \\
\hline TUG (sec) & $22.13 \pm 15.60$ & $20.02 \pm 12.80$ & 0.03 & 0.15 \\
FRT (cm) & $17.3 \pm 7.6$ & $18.0 \pm 7.1$ & 0.51 & 0.10 \\
\hline
\end{tabular}

Values are presented as mean \pm standard deviation.

TUG, Timed Up and Go test; FRT, Functional Reach Test. posturography measures $(P>0.05)$.

\section{DISCUSSION}

This study found that the direct focal vibratory stimuli at 100 $\mathrm{Hz}$ applied to the plantar region for $5 \mathrm{~min}$ in a single session improved functional mobility and dynamic balance as measured by the TUG, plantarflexor spasticity, and ankle PROM in a sample of patients poststroke.

Vibratory stimulation is a simple, noninvasive therapeutic method for the treatment of motor disorders and to reduce spasticity. To facilitate the paretic muscle, application of vibration to the muscle selectively stimulates the Ia or primary spindle endings and Ia inhibitory interneurons which causes a tonic vibration reflex thereby induce nonvoluntary contraction in the vibrated muscle and inhibits the muscle antagonistic to the activated muscle. Therefore, vibratory stimulation is traditionally applied to the antagonistic muscles in order to decrease the tone of spastic muscles (Bishop, 1975).

However, it was found in this study that sensory vibratory stimulation applied to the plantar region of adult post stroke improved plantarflexor spasticity and ankle PROM; both important factors for balance and fall risk. A 1-point reduction in the median MMAS score suggests that the immediate antispastic effects of vibration applied to the plantar region have clinical importance. Improvement in spasticity level found in this study is consistent with those in the literature that concluded focal vibration could be used to reduce spasticity in neurological conditions (KarimiAhmadAbadi et al., 2017; Murillo et al., 2014). The reason why the direct application of vibratory stimulation to the plantar region reduced the spasticity of the plantarflexor muscles remains to be elucidated. The antispastic effect observed following vibration applied to the sole region in this sample of patients poststroke might be explained by central mechanisms (Binder et al., 2009; Nardone and Schieppati, 2005). Vibration applied to the plantar

Table 3. Results of posturography

\begin{tabular}{lccccc}
\hline \multirow{2}{*}{ Measure } & \multicolumn{2}{c}{ Eyes open } & & \multicolumn{2}{c}{ Eyes closed } \\
\cline { 2 - 3 } \cline { 5 - 6 } & Pre & Post & & Pre & Post \\
\hline $\begin{array}{c}\text { Displacement velocity } \\
(\mathrm{cm} / \mathrm{sec})\end{array}$ & $3.03 \pm 0.51$ & $2.98 \pm 0.45$ & & $3.39 \pm 0.48$ & $3.32 \pm 0.43$ \\
ML displacement $(\mathrm{cm})$ & $0.33 \pm 0.12$ & $0.36 \pm 0.18$ & & $0.39 \pm 0.17$ & $0.39 \pm 0.16$ \\
AP displacement $(\mathrm{cm})$ & $0.48 \pm 0.12$ & $0.41 \pm 0.20$ & & $0.64 \pm 0.17$ & $0.66 \pm 0.22$ \\
Surface area $\left(\mathrm{cm}^{2}\right)$ & $4.18 \pm 5.73$ & $3.55 \pm 2.48$ & & $4.54 \pm 2.80$ & $5.10 \pm 3.70$ \\
\hline
\end{tabular}

Values are presented as mean \pm standard deviation $\mathrm{ML}$, medial-lateral; AP, anterior-posterior. 
region could enhance the inhibition of neuronal elements in the nonvibrated plantarflexor muscles at a supraspinal level and decrease the excitability of the alpha motor neuron. The decreases of spasticity may be explained by the duration of vibration. Vibration for more than $1 \mathrm{~min}$ has been reported to reduce motor unit firing, motor output and cortical excitability (Issurin, 2005).

Balance is influenced by multiple factors. Normal muscle tone and sufficient ankle ROM are two important factors that allow the individual to have balance and postural stability during gait. Upper motor neuron syndrome such as stroke, can result in disturbed motor control and joint immobilization which can gradually lead to soft tissue contracture and increased muscle stiffness (Gracies, 2005). Hyperactive stretch reflexes together with increased passive stiffness due to mechanical changes in muscles, if untreated, can worsen the condition. Spastic muscle fibers have been demonstrated to be almost twice as stiff when compared to normal fibers (Fridén and Lieber, 2003). Plantarflexor muscle stiffness has been also reported to be abnormally high on the paretic side (Lamontagne et al., 2002).

In the present study, patients had limited ankle PROM indicating soft tissue and muscle contracture around the ankle joint. Although ankle PROM showed improvement after the application of vibration to the foot plantar region and large effect size was obtained, it was still limited. It remains to be investigated whether applying vibration to the plantar region for longer time duration or performing multiple treatment sessions may result in greater increases in ankle PROM. Improvement in ankle PROM obtained in this study that is consistent with Karimi-AhmadAbadi et al. (2017) might be explained by spasticity reduction as well as an increase in tissue compliance (Bae and Kim, 2012).

The FRT assesses the ability of patients to reach forward and can help to identify fall risk. There was not a significant improvement in the FRT after treatment despite increases in reaching function $(\sim 4 \%)$. The FRT values found in the prepost time points indicate that patients in this study had limited functional balance being in the moderate risk category for falls (Duncan et al., 1992). The nonsignificant result might be attributable to sample size or inadequate dose of vibration. A long-term study with a larger sample of participants and longer duration of vibration may be warranted. Nevertheless, a study found that FRT is influenced by trunk movement, does not measure all aspects of balance, the FR distance is not correlated with center of mass displacement, and it is a weak measure for evaluation of stability limits (Jonsson et al., 2003).

A significant, small effect on the TUG test was identified. The
TUG test is a measure of mobility and dynamic balance that improved about $10 \%$ after the intervention. Improvement in the TUG test might be explained by decreases in spasticity level, increased ankle PROM, and perhaps enhanced sensory information provided by vibration. Although a $10 \%$ reduction in the mean TUG test score may be clinically acceptable and relevant following a single treatment applied for $5 \mathrm{~min}$, a study reported a change of at least $23 \%$ in TUG score must be observed poststroke to indicate a real, clinical improvement (Flansbjer et al., 2005). Small sample size, single treatment session, and duration of vibration for 5 min might be the reasons for the less than optimal changes that occurred in TUG test. Mean TUG scores pre- and posttreatment (>20 sec) indicate that the participants still need further intervention (Podsiadlo and Richardson, 1991). In the present study, the participants were also tested with a force plate whereby the body sway and the static postural control could be objectively evaluated. Both static and dynamic balances are important for the performance of daily activities. Similar to the FRT, posturography (i.e., a static measure of balance) did not show significant changes. Static balance is important as it is a measure to predict independence and fall risk (Rogers et al., 2003). The objective data provided by the force plate indicated that no changes occurred with the vibration protocol used in this study. This indicates that the strategies and neuromuscular responses to minimize the COP displacements did not occur to improve balance in static posture (Visser et al., 2008). However, in a study with women 60 years or older investigating the effect of vibratory stimuli of $100 \mathrm{~Hz}$ applied to the plantar region for 12 sessions, found beneficial effects in posturography measures in anterior displacement, postural control of the AP axis, and the eyes closed condition. No effects were identified in the clinical balance tests of OLS, FRT, and TUG test (Wanderley et al., 2011). Further, in a recent study in patients with stroke, the plantar vibration improved the balance in terms of Mini-BESTest scores (Karimi-AhmadAbadi et al., 2017).

In eyes closed, the postural sway was higher compared to the eyes open reflected in the significant main effects for mean velocity, AP and ML directions as well as high values detected for surface area during eyes closed. These findings consistent with previous report (Marigold and Eng, 2006) indicate that the eyes closed condition challenged the postural stability, and postural control in both sagittal and frontal planes decreased following changes in test condition from eyes open to eyes closed. The worst performance in eyes closed and decrease in balance control has been reported in healthy participants, as well (Sawacha et al., 2013). This finding indicates that visual information along with the informa- 
tion from somatosensory and vestibular systems is important to maintain balance (De Oliveira et al., 2008; Horak et al., 1997) and removing information from eyes increases the body sway even in healthy participants and in quiet standing (Sawacha et al., 2013). However, patients with stroke have been demonstrated to have poor balance in both eyes open and eyes closed, and visual information do not improve the postural control and balance compared to the healthy participants (Geurts et al., 2005; Sawacha et al., 2013).

The present study has several limitations. First, the number of participants was small. Second, only functional assessment tools for balance were used in this study, thus future studies should include system based tools such as the BESTest (Horak et al., 2009) which can help clinicians to specify the type of balance dysfunction in order to direct treatment and to determine what system may be influenced by vibration (Mancini and Horak, 2010). Third, no control or placebo group was included in the study to compare the post measures. A control group was not included in this study because it was assumed that the placebo vibration would not show significant better effects on balance compared to the real vibration. Fourth, the assessor and the physiotherapist treating the participants were the same, introducing possible bias. Finally, participants were not followed-up to determine the longterm effects.

In conclusion, vibratory stimuli at a frequency of $100 \mathrm{~Hz}$ applied to the plantar region of patients poststroke could have beneficial effects on muscle spasticity assessed by MMAS, ankle PROM measured by goniometer, and dynamic balance assessed by TUG test. With regard to static balance, there were not significant improvements. Further research is necessary to characterize the effects and the optimal dose using a rigorous, double-blinded randomized controlled study design in a larger sample of participants. Based on the results, the vibration stimuli applied to the plantar region of the more affected foot may be added to PT program to improve functional mobility and dynamic balance in patients with stroke.

\section{CONFLICT OF INTEREST}

No potential conflict of interest relevant to this article was reported.

\section{ACKNOWLEDGMENTS}

The authors would like to thank the patients for participation in this study and the Research Deputy, Tehran University of Medical Sciences (TUMS) for their support.

\section{REFERENCES}

Ansari NN, Adelmanesh F, Naghdi S, Tabtabaei A. The effect of physiotherapeutic ultrasound on muscle spasticity in patients with hemiplegia: a pilot study. Electromyogr Clin Neurophysiol 2006a;46:247-252.

Ansari NN, Naghdi S, Arab TK, Jalaie S. The interrater and intrarater reliability of the Modified Ashworth Scale in the assessment of muscle spasticity: limb and muscle group effect. NeuroRehabilitation 2008;23: 231-237.

Ansari NN, Naghdi S, Moammeri H, Jalaie S. Ashworth Scales are unreliable for the assessment of muscle spasticity. Physiother Theory Pract 2006b;22:119-125.

Arene N, Hidler J. Understanding motor impairment in the paretic lower limb after a stroke: a review of the literature. Top Stroke Rehabil 2009; 16:346-356.

Bae SH, Kim KY. The effect of vibratory stimulation on tissue compliance and muscle activity in elbow flexor spasticity. J Phys Ther Sci 2012;24: 751-754.

Binder C, Kaya AE, Liepert J. Vibration prolongs the cortical silent period in an antagonistic muscle. Muscle Nerve 2009;39:776-780.

Bishop B. Vibratory stimulation. Part III. Possible applications of vibration in treatment of motor dysfunctions. Phys Ther 1975;55:139-143.

Carey LM. Somatosensory loss after stroke. Crit Rev Phys Rehabil Med 1995;7:51-91.

Chen JC, Shaw FZ. Progress in sensorimotor rehabilitative physical therapy programs for stroke patients. World J Clin Cases 2014;2:316-326.

De Oliveira CB, de Medeiros IR, Frota NA, Greters ME, Conforto AB. Balance control in hemiparetic stroke patients: main tools for evaluation. J Rehabil Res Dev 2008;45:1215-1226.

Divani AA, Vazquez G, Barrett AM, Asadollahi M, Luft AR. Risk factors associated with injury attributable to falling among elderly population with history of stroke. Stroke 2009;40:3286-3292.

Duncan P, Studenski S, Richards L, Gollub S, Lai SM, Reker D, Perera S, Yates J, Koch V, Rigler S, Johnson D. Randomized clinical trial of therapeutic exercise in subacute stroke. Stroke 2003;34:2173-2180.

Duncan PW, Studenski S, Chandler J, Prescott B. Functional reach: predictive validity in a sample of elderly male veterans. J Gerontol 1992; 47:M93-98.

Duncan PW, Weiner DK, Chandler J, Studenski S. Functional reach: a new clinical measure of balance. J Gerontol 1990;45:M192-197.

Flansbjer UB, Holmbäck AM, Downham D, Patten C, Lexell J. Reliability of gait performance tests in men and women with hemiparesis after 
stroke. J Rehabil Med 2005;37:75-82.

Fridén J, Lieber RL. Spastic muscle cells are shorter and stiffer than normal cells. Muscle Nerve 2003;27:157-164.

Geiger RA, Allen JB, O'Keefe J, Hicks RR. Balance and mobility following stroke: effects of physical therapy interventions with and without biofeedback/forceplate training. Phys Ther 2001;81:995-1005.

Geurts AC, de Haart M, van Nes IJ, Duysens J. A review of standing balance recovery from stroke. Gait Posture 2005;22:267-281.

Ghotbi N, Nakhostin Ansari N, Naghdi S, Hasson S. Measurement of lower-limb muscle spasticity: intrarater reliability of Modified Modified Ashworth Scale. J Rehabil Res Dev 2011;48:83-88.

Gracies JM. Pathophysiology of spastic paresis. I: Paresis and soft tissue changes. Muscle Nerve 2005;31:535-551.

Horak FB, Henry SM, Shumway-Cook A. Postural perturbations: new insights for treatment of balance disorders. Phys Ther 1997;77:517-533.

Horak FB, Wrisley DM, Frank J. The Balance Evaluation Systems Test (BESTest) to differentiate balance deficits. Phys Ther 2009;89:484-498.

Issurin VB. Vibrations and their applications in sport. A review. J Sports Med Phys Fitness 2005;45:324-336.

Johansson BB, Haker E, von Arbin M, Britton M, Långström G, Terént A, Ursing D, Asplund K; Swedish Collaboration on Sensory Stimulation After Stroke. Acupuncture and transcutaneous nerve stimulation in stroke rehabilitation: a randomized, controlled trial. Stroke 2001;32: 707-713.

Jonsson E, Henriksson M, Hirschfeld H. Does the functional reach test reflect stability limits in elderly people? J Rehabil Med 2003;35:26-30.

Karimi-AhmadAbadi A, Naghdi S, Nakhostin Ansari N, Fakhari Z, Khalifeloo M. A clinical single blind study to investigate the immediate effects of plantar vibration on balance in patients after stroke. J Bodyw Mov Ther 2017 May 5 [Epub]. https://doi.org/10.1016/j.jbmt.2017.04. 013.

Knorr S, Brouwer B, Garland SJ. Validity of the Community Balance and Mobility Scale in community-dwelling persons after stroke. Arch Phys Med Rehabil 2010;91:890-896.

Lamb SE, Ferrucci L, Volapto S, Fried LP, Guralnik JM; Women's Health and Aging Study. Risk factors for falling in home-dwelling older women with stroke: the Women's Health and Aging Study. Stroke 2003;34: 494-501.

Lamontagne A, Malouin F, Richards CL, Dumas F. Mechanisms of disturbed motor control in ankle weakness during gait after stroke. Gait Posture 2002;15:244-255.

Lu J, Xu G, Wang Y. Effects of whole body vibration training on people with chronic stroke: a systematic review and meta-analysis. Top Stroke Rehabil 2015;22:161-168.

Mancini M, Horak FB. The relevance of clinical balance assessment tools to differentiate balance deficits. Eur J Phys Rehabil Med 2010;46:239248.

Marigold DS, Eng JJ. The relationship of asymmetric weight-bearing with postural sway and visual reliance in stroke. Gait Posture 2006;23:249255.

Murillo N, Valls-Sole J, Vidal J, Opisso E, Medina J, Kumru H. Focal vibration in neurorehabilitation. Eur J Phys Rehabil Med 2014;50:231242.

Naghdi S, Khalifeloo M, Nakhostin Ansarin N, Akbari M, Jalaie S, Jannat $D$. The short-term effects of plantar vibration on balance disorder after stroke. Audiology 2013;22:104-109.

Nakhostin Ansari N, Naghdi S, Forogh B, Hasson S, Atashband M, Lashgari E. Development of the Persian version of the Modified Modified Ashworth Scale: translation, adaptation, and examination of interrater and intrarater reliability in patients with poststroke elbow flexor spasticity. Disabil Rehabil 2012;34:1843-1847.

Nardone A, Schieppati M. Reflex contribution of spindle group Ia and II afferent input to leg muscle spasticity as revealed by tendon vibration in hemiparesis. Clin Neurophysiol 2005;116:1370-1381.

$\mathrm{Ng}$ SS, Hui-Chan CW. The timed up \& go test: its reliability and association with lower-limb impairments and locomotor capacities in people with chronic stroke. Arch Phys Med Rehabil 2005;86:1641-1647.

Parsons SL, Mansfield A, Inness EL, Patterson KK. The relationship of plantar cutaneous sensation and standing balance post-stroke. Top Stroke Rehabil 2016;23:326-332.

Podsiadlo D, Richardson S. The timed "Up \& Go": a test of basic functional mobility for frail elderly persons. J Am Geriatr Soc 1991;39:142148.

Pollock A, Baer G, Campbell P, Choo PL, Forster A, Morris J, Pomeroy VM, Langhorne P. Physical rehabilitation approaches for the recovery of function and mobility following stroke. Cochrane Database Syst Rev 2014;(4):CD001920.

Pollock A, Baer G, Pomeroy V, Langhorne P. Physiotherapy treatment approaches for the recovery of postural control and lower limb function following stroke. Cochrane Database Syst Rev 2003;(2):CD001920.

Pomeroy VM, King L, Pollock A, Baily-Hallam A, Langhorne P. Electrostimulation for promoting recovery of movement or functional ability after stroke. Cochrane Database Syst Rev 2006;(2):CD003241.

Priplata AA, Niemi JB, Harry JD, Lipsitz LA, Collins JJ. Vibrating insoles and balance control in elderly people. Lancet 2003;362:1123-1124.

Radinmehr H, Nakhostin Ansari N, Naghdi S, Olyaei G, Tabatabaei A. Effects of one session radial extracorporeal shockwave therapy on post-stroke plantarflexor spasticity: a single-blind clinical trial. Disabil Rehabil 2017;39:483-490.

Rogers ME, Rogers NL, Takeshima N, Islam MM. Methods to assess and 
improve the physical parameters associated with fall risk in older adults. Prev Med 2003;36:255-264.

Sawacha Z, Carraro E, Contessa P, Guiotto A, Masiero S, Cobelli C. Relationship between clinical and instrumental balance assessments in chronic post-stroke hemiparesis subjects. J Neuroeng Rehabil 2013; 10:95.

Schlee G, Reckmann D, Milani, TL. Whole body vibration training reduces plantar foot sensitivity but improves balance control of healthy participants. Neurosci Lett 2012;506:70-73.

Sterzi R, Bottini G, Celani MG, Righetti E, Lamassa M, Ricci S, Vallar G. Hemianopia, hemianaesthesia, and hemiplegia after right and left hemisphere damage. A hemispheric difference. J Neurol Neurosurg Psychiatry 1993;56:308-310.
Sui J, Shull P, Ji L. Pilot study of vibration stimulation on neurological rehabilitation. Biomed Mater Eng 2014;24:2593-2601.

Tyson SF, Hanley M, Chillala J, Selley A, Tallis RC. Balance disability after stroke. Phys Ther 2006;86:30-38.

Visser JE, Carpenter MG, van der Kooij H, Bloem BR. The clinical utility of posturography. Clin Neurophysiol 2008;119:2424-2436.

Wanderley FS, Alburquerque-Sendín F, Parizotto NA, Rebelatto JR. Effect of plantar vibration stimuli on the balance of older women: a randomized controlled trial. Arch Phys Med Rehabil 2011;92:199-206.

Yates JS, Lai SM, Duncan PW, Studenski S. Falls in community-dwelling stroke survivors: an accumulated impairments model. J Rehabil Res Dev 2002;39:385-394. 